\title{
Longstanding Hypoparathyroidism in a Fifty-Two-Year-Old Woman Misdiagnosed as Spondyloarthropathy
}

\author{
Mozhdeh Zabihiyeganeh ${ }^{1}$; Seyed Adel Jahed ${ }^{2, *} ;$ Hounaz Akbari $^{1}$ \\ ${ }^{1}$ Firouzgar General Hospital, Iran University of Medical Sciences, Tehran, IR Iran

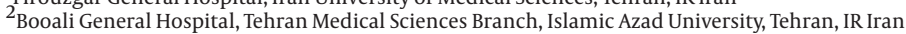 \\ *Corresponding Author: Seyed Adel Jahed, Booali General Hospital, Tehran Medical Sciences Branch, Islamic Azad University, Tehran, IR Iran. Tel: +98-2122770651, Fax: +98-2122770699, \\ E-mail: adeljahed@irimc.org
}

Received: August 6, 2014; Revised: September 27, 2014; Accepted: October 12, 2014

\begin{abstract}
Introduction: Hypoparathyroidism might cause various musculoskeletal findings, resembling Spondyloarthropathies.
Case Presentation: We described a 52-year-old woman, diagnosed as a case of undifferentiated spondyloarthropathy for ten years, who was unresponsive to classic anti-inflammatory therapies. She developed anterior ischemic optic neuropathy and had elevated muscle enzymes during the course of disease. On a routine checkup, marked hypocalcemia and suppressed parathyroid hormone levels were found. Calcium and calcitriol therapy led to clinical and laboratory improvement.

Conclusions: This case emphasizes the importance of recognizing rheumatic manifestations of hypoparathyroidism to preclude unnecessary treatments. Serum calcium should be included in the diagnostic workup of these patients.
\end{abstract}

Keywords: Hypoparathyroidism; Anterior Ischemic Optic Neuropathy; Spondyloarthropathy

\section{Introduction}

Hypoparathyroidism with low parathyroid hormone (PTH) occurs when secretion of PTH decreases due to destruction, abnormal development, or altered regulation of parathyroid gland. The most common cause of hypoparathyroidism is surgery, i.e. thyroidectomy. Clinical manifestations are often related to hypocalcemia and hyperphosphatemia whereas musculoskeletal features and rarely, radiologic changes suggestive of spondyloarthropathy might be seen (1-3). We hereby reported a case of hypoparathyroidism that was misdiagnosed as refractory spondyloarthropathy and therefore, had been treated with anti-tumor necrosis factor (anti-TNF).

\section{Case Presentation}

In April 2011, a 50-year-old woman was referred to our outpatient Rheumatology Clinic in Firouzgar General Hospital, Tehran, Iran, with 10 years history of progressive neck and back inflammatory pain suggestive of undifferentiated spondyloarthropathy. She had a history of surgical thyroidectomy at the age of fifteen, an episode of seizure at the age of sixteen, and bilateral cataracts extraction at forty. On physical examination, she had typical ankylosing spondylitis posture with decreased spinal mobility, limited neck rotation, and restricted internal hip rotation but near normal chest expansion. Result of modified Schober's test was $13 \mathrm{~cm}$ and Fabere test result was positive. Results of Trousseaus and Chvostek's signs were both negative. Laboratory findings revealed elevated ESR ( $65 \mathrm{~mm} / \mathrm{h})$ and CRP (219 nmol/L). Results of complete blood count, blood glucose, urea, creatinine, and liver and thyroid function tests were within normal limits. Results of anti-nuclear antibodies, rheumatoid factor, anti-CCP, and HLA-B27 tests were negative. In skeletal survey, pelvic radiography showed ossification of iliolumbar ligaments at lateral margin of acetabulum and capsular calcification around hip joints. Enthesopathic changes were seen at muscular insertions of both trochanters, ischial tuberosities, and iliac crests. Computed tomography (CT) of Pelvic revealed normal sacroiliac joints but osteophytes at inferior margins of the joint (Figure 1). Using dual-energy x-ray absorptiometry (DEXA) method, a calibrated Norland bone densitometer (Model XR 600, Norland, Wisconsin, USA) showed elevated bone mineral density at lumbar spine and total femur regions with $\mathrm{T}$ scores of +4.8 and +1.4 , respectively. A plain CT scan of brain showed bilateral symmetric basal ganglia calcifications (Table 1).

Raised inflammatory markers (ESR and CRP) concomitant with chronic inflammatory back pain were suggestive of an atypical spondyloarthropathy, which is more common in women. Indometacin and sulfasalazine were started but she did not achieve favorable response. Lowdose oral prednisolone was intermittently used for treating inflammatory attacks. Supplemental calcium and vitamin D were concomitantly used. Two years later, she 


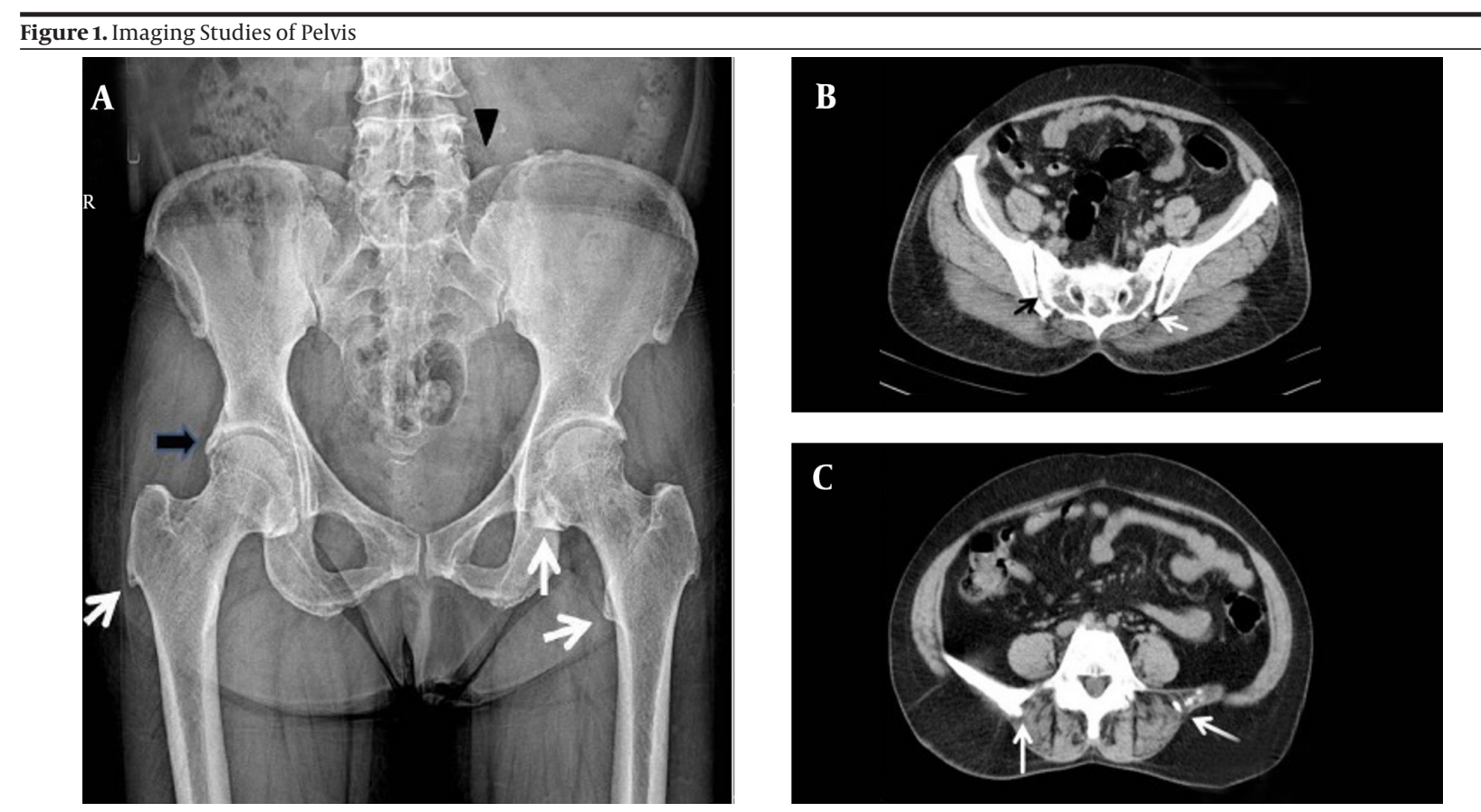

A, (Left) Anteroposterior radiograph of pelvis shows mineralization of iliolumbar ligament (black arrowhead), ossification at the lateral margin of acetabulum (black arrow), osseous proliferation of lesser and greater trochanters, and capsular calcification of the hip joint (white arrows). B, (Right-top) Axial view in computed tomography shows a normal sacroiliac joint (black arrow) but bridging calcification at the inferior margin of the joint (white arrow). C, (Right-bottom) Axial computed tomography shows ossification of the iliolumbar ligament (white arrows).

\begin{tabular}{|c|c|}
\hline Parameter & Values \\
\hline FBS, $\mathrm{mmol} / \mathrm{L}$ & 5 \\
\hline $\mathrm{Cr}, \mu \mathrm{mol} / \mathrm{L}$ & 62 \\
\hline WBC, cell/L & $6.5 \times 10^{9}$ \\
\hline $\mathrm{HB}, \mathrm{g} / \mathrm{L}$ & 110 \\
\hline Calcium, $\mathrm{mmol} / \mathrm{L}$ & 1.38 \\
\hline Albumin, $\mathbf{g} / \mathbf{L}$ & 40 \\
\hline Phosphorus, mmol/L & 2.26 \\
\hline PTH, ng/L & $<4$ \\
\hline 25(OH)D, nmol/L & 80 \\
\hline ALT, $\mu$ kat $/ \mathbf{L}$ & 0.20 \\
\hline AST, $\mu$ kat $/ \mathbf{L}$ & 0.42 \\
\hline CPK, $\mu$ kat $/ \mathrm{L}$ & 34 \\
\hline LDH, $\mu$ kat/L & 20 \\
\hline ESR, $\mathbf{m m} / \mathbf{h}$ & 65 \\
\hline CRP, nmol/L & 219 \\
\hline TSH, mIU/L & 2 \\
\hline HLA B27 & Negative \\
\hline Anti-nuclear antibody & Negative \\
\hline Pelvic Radiography & Ossification of iliolumbar ligament \\
\hline Pelvic CT scan & Normal sacroiliac joints \\
\hline Brain CT scan & Bilateral basal ganglia calcifications \\
\hline \multicolumn{2}{|c|}{ Bone densitometry ( $\mathrm{T}$ score) } \\
\hline Total Femur & +1.4 \\
\hline Lumbar & +4.8 \\
\hline
\end{tabular}


developed blurred vision, which was diagnosed as anterior ischemic optic neuropathy (AION) by the consultant ophthalmologist. High-dose oral prednisolone $(1 \mathrm{mg} / \mathrm{kg})$ led no improvement; therefore, infliximab ( $5 \mathrm{mg} / \mathrm{kg}$ ) was added to steroid therapy and led to dramatic response after two courses, two weeks apart. On admission for receiving infliximab, biochemical assessment revealed severe hypocalcemia $(1.38 \mathrm{mmol} / \mathrm{L})$, hyperphosphatemia (2.26 mmol/L), undetectable PTH $(<4 \mathrm{ng} / \mathrm{L})$, but normal serum 25-hydroxy vitamin D (80 nmol/L), albumin, alanine transaminase, and aspartate transaminase. Elevated creatine phosphokinase (CPK) and lactate dehydrogenase (LDH) were reported (34 and $20.04 \mu \mathrm{kat} / \mathrm{L}$, respectively). Electromyography and echocardiography findings were normal. Classic treatment of hypoparathyroidism with elemental calcium ( $2 \mathrm{~g} /$ day) and calcitriol ( $1 \mu \mathrm{g} /$ day) led to clinical and laboratory improvement.

\section{Discussion}

We reported an unusual case of hypoparathyroidism that was misdiagnosed and initially managed as spondyloarthropathy. To our knowledge, there were only a few published cases of hypoparathyroidism that instead of presenting common manifestations of hypocalcemia, had presented with clinical and radiologic features suggestive of spondyloarthropathies. In this case, chronic development of postsurgical hypocalcemia might explain the absence of acute signs and symptoms of hypocalcemia. The significant strength of this case could be the occurrence of three rare presentations of hypoparathyroidism in a single case, which misled the clinicians to an incorrect diagnosis and unnecessary treatments: unusual rheumatologic manifestations in the absence of clinical hallmarks of hypocalcemia, elevated muscle enzymes, and optic neuropathy. Radiologic and clinical findings of our case were suggestive of undifferentiated spondyloarthropathy, which was similar to previously reported cases (1-6). Increased bone density in our case was quite consistent with the published cases (7). The raised serum CPK level, found in this patient, has been rarely reported in chronic hypocalcemia. Increased permeability of muscle membrane, induced by hypocalcemia, is the most accepted explanation for CPK release $(8,9)$. The occurrence of sight-threatening hypocalcemia-associated AION, as a rare but eminently treatable cause of papilledema, forced us to use infliximab. In the majority of such cases, high local cerebrospinal fluid (CSF) pressure on the head of optic nerve has been postulated as the cause of ischemic neuronal loss; however, in the minorities with normal CSF pressure, hypocalcemia itself might cause the localized axonal and optic disc swelling resulting in AION (10). Paying more attention to some noticeable points including female sex, absence of sacroiliitis, HLA-B27 negativity, and clear clues of hypocalcemia such as vague neuromuscular symptoms, early onset cataract, convulsion, and history of thyroidectomy could lead to early differentiation of the case from spondyloarthropathies. Elevated serum muscular enzymes might be considered as a marker of a possible inflammatory myopathic process. Although the absence of muscular pathology could be measured as a limitation in this report, in fact, the presence of typical inflammatory back pain, normal electromyography findings, and dramatic decline of serum CPK and LDH following treatment with calcium and calcitriol dismissed performing a muscle biopsy. This case emphasizes on considering calcium disorders in patients with features of atypical spondyloarthropathy. Spondyloarthropathy along with vague neuromuscular complains in the presence of hypocalcemia should raise the possibility hypoparathyroidism. In such rather rare cases, hazards of unnecessary heavy rheumatologic treatments could be avoided using a simple, low-cost, and fast-responding treatment with calcium and vitamin D metabolites.

\section{Acknowledgements}

We would like to thank Dr. Zahra Beiki.

\section{Authors' Contributions}

Dr. Mozhdeh Zabihiyeganeh: Case finding, main idea, writing, and submission of manuscript; Dr. Seyed Adel Jahed: Writing, discussion, and editing manuscript; and Dr. Akbari: Writing the manuscript.

\section{References}

1. Korkmaz C, Yasar S, Binboga A. Hypoparathyroidism simulating ankylosing spondylitis. Joint Bone Spine. 2005;72(1):89-91.

2. Ibn Yacoub Y, Rostom S, Hajjaj-Hassouni N. Uncommon case of ankylosing spondylitis associated with spontaneous occurring hypoparathyroidism. Rheumatol Int. 2011;31(5):681-3.

3. Goswami R, Ray D, Sharma R, Tomar N, Gupta R, Gupta N, et al. Presence of spondyloarthropathy and its clinical profile in patients with hypoparathyroidism. Clin Endocrinol (Oxf). 2008;68(2):258-63.

4. Unverdi S, Ozturk MA, Inal S, Selek H, Goker B, Haznedaroglu C, et al. Idiopathic hypoparathyroidism mimicking diffuse idiopathic skeletal hyperostosis. J Clin Rheumatol. 2009;15(7):361-2.

5. Okazaki T, Takuwa Y, Yamamoto M, Matsumoto T, Igarashi T, Kurokawa T, et al. Ossification of the paravertebral ligaments: a frequent complication of hypoparathyroidism. Metabolism. 1984;33(8):710-3.

6. Jakkani RK, Sureka J, Mathew J. Spondyloarthropathy occurring in long standing idiopathic hypoparathyroidism. Radiol Case Rep. 2011;6(4):545.

7. Laway BA, Goswami R, Singh N, Gupta N, Seith A. Pattern of bone mineral density in patients with sporadic idiopathic hypoparathyroidism. Clin Endocrinol (Oxf). 2006;64(4):405-9.

8. Syriou V, Kolitsa A, Pantazi L, Pikazis D. Hypoparathyroidism in a patient presenting with severe myopathy and skin rash. Case report and review of the literature. Hormones (Athens). 2005;4(3):161-4.

9. Kajitani TR, Silva RV, Bonfa E, Pereira RM. Hypoparathyroidism mimicking ankylosing spondylitis and myopathy: a case report. Clinics (Sao Paulo). 2011;66(7):1287-90.

10. Goyal JL, Kang J, Gupta R, Anand A, Arora R, Jain P. Bilateral Papilledema in Hypocalcemia. Del J Ophthalmol. 2012;23(2):127-30. 